\title{
Rapid responses in bovine milk fatty acid composition and phenol content to various tanniferous forages
}

\author{
A. Birkinshaw ${ }^{1}$, A. Schwarm² ${ }^{2}$ S. Marquardt ${ }^{3}$, M. Kreuzer ${ }^{1}$ and M. Terranova ${ }^{4,5}$ \\ ${ }^{1}$ ETH Zurich, Institute of Agricultural Sciences, Universitätstrasse 2, 8092 Zurich, Switzerland \\ ${ }^{2}$ Norwegian University of Life Sciences, Department of Animal and Aquacultural Sciences Arboretveien 6, 1433 Ås, Norway \\ ${ }^{3}$ International Livestock Research Institute (ILRI), Mazingira Centre, Nairobi 00100, Kenya \\ ${ }^{4}$ ETH Zurich, AgroVet-Strickhof, Eschikon 27, 8315 Lindau, Switzerland
}

KEY WORDS: blackcurrant, grape vine, hazel, rosebay willow, silver birch, wood avens

Received: $\quad 4$ August 2020

Revised: $\quad 20$ October 2020

Accepted: 30 November 2020

${ }^{5}$ Corresponding author:

e-mail: melissa-terranova@ethz.ch

\begin{abstract}
Milk and dairy products considerably contribute to the nutritional value of human diets. In order to benefit human nutrition bovine milk fatty acid composition and phenol content are effectively manipulated by the cow's diet. However, response times taken for these alterations to occur have not been quantified. In the present study, fatty acid composition and phenol content of the milk were evaluated after three days of feeding six cows six different diets, supplemented with six different tanniferous plants (hazel, silver birch, blackcurrant, grape vine, wood avens and rosebay willow with total tannin concentrations of $26,36,42,52,55$ and $79 \mathrm{~g} / \mathrm{kg}$ dry matter, respectively). Lucerne was applied as the low-phenol control diet. Substantial changes in total phenols and fatty acids were found in milk samples after just three days. Proportions of cis-9 trans-11 C18:2 and trans-11 C18:1 declined by 29 and 68\%, respectively, in comparison to milk from cows fed lucerne, indicating a definitive ruminal biohydrogenation response. However, there were no significant effects between test plants and lucerne when comparing C18:3 $n-3$ and C18:2 n-6 proportions in milk fat. So, it was demonstrated that phenols and certain individual fatty acids in bovine milk can be rapidly modified by adding specific tanniferous plants to the diet.
\end{abstract}

\section{Introduction}

Bovine milk and dairy products are widely consumed and routinely considered staples in many countries. Some dietary phenolic compounds are transferrable to bovine milk and may exert benefits for human health. Recognised benefits include protection against oxidative damage (Alenisan et al., 2017), prevention of chronic diseases (Vuazour et al., 2010) and inhibition of oxidative stress and cell destruction associated with degenerative diseases and with modern lifestyles (Willcox et al., 2004). Additionally, antioxidant effects of phenols help prevent oxidative scavenging of free radicals in dairy products, prolonging shelf life (Alenisan et al., 2017). Also, specific fatty acids (FA) have been clinically documented to have particular health promoting effects. These include $\alpha$-linolenic acid (C18:3 n-3; ALA) and rumenic acid (cis-9, trans-11 C18:2; RA) one of the notably beneficial conjugated linoleic acid isomers caused by ruminal biohydrogenation (Białek et al., 2017). Benefits include decreased inflammation, prevention of a number of diseases, optimal cell signalling and increased immune function (German and Dillard, 2006; Białek et al., 2017). Other FA such as linoleic acid (C18:2 n-6; LA), are explicit in modern diets and may cause adverse effects when consumed in disproportionate amounts (Calder, 2015). 
Milk fat is one of the most dynamic natural fats. It contains $>400$ individual FAs and is continuously changed according to internal and external factors including lactation, farm management systems, seasonality and feeding strategies (Månsson, 2008). Routine methods for dietary manipulation of the FA composition of bovine milk include varying dietary carbohydrate profiles thus influencing de novo FA synthesis or by including different types of dietary fat (including rumen-protected oils; Petit et al., 2002) and phenolic compounds in the diet. Phenols, particularly tannins, may counteract or inhibit ruminal biohydrogenation (RBH) of polyunsaturated FA (PUFA) such as ALA and LA (Palmquist and Jenkins, 2017; Hanuš et al., 2018; Majewska and Kowalik, 2020). Tannins are commonly divided into two main classes with differing effects on RBH. Insoluble condensed tannins (CT) have a very high molecular weight. They are formed by the polymerisation of flavon3-ols and have an inhibitory effect on RBH (Vasta et al., 2019). The soluble hydrolysable tannins are non-flavonoid compounds that also seem to have a modulatory effect on RBH (Vasta et al., 2019). The exact mechanisms by which tannins inhibit microbes involved in $\mathrm{RBH}$ remain ambiguous; however, tannic effects are known to be modulated by dose, chemical composition, macronutrient interaction and potential ruminal microbiota adaption to these phytochemicals (Morales and Ungerfeld, 2015; Vasta et al., 2019). The results of both in vitro and in vivo studies suggest that rumen bacteria involved in lipolysis, the first step of RBH and specifically the conversion of ALA and LA to vaccenic acid (VA), and VA to C18:0, are affected differently by tannins (Vasta et al., 2019). One theory is that tannins may exert toxic effects by altering the permeability of bacterial membranes (Frutos et al., 2004). Other theories suggest that tannins reduce RBH through the inhibition of the activity of the rumen microbes rather than through the inhibition of their enzymatic activity and that this inhibition is not constant but affects the different steps of RBH at different rates (Vasta et al., 2009).

Ruminant milk composition has been positively influenced by the addition of specific dietary supplements in vivo - including grapeseed extract, oilseeds, vegetable oils and rumen-protected fats - by favourably altering phenol contents and FA composition (Singh et al., 2018; Castro et al., 2019; Leparmarai et al., 2019). Denninger et al. (2020) recently demonstrated significant changes in the milk FA composition within 1 to 3 days of cows re- ceiving a tanniferous Acacia mearnsii bark extract. As dietary supplements are often expensive, in limited supply and may decrease palatability, naturally occurring feed components are of particular interest in ruminant nutrition. These may include biodiverse pasture and regional forages that often contain tannin-rich plants. Rapid responses of the milk FA profile and phenol content to these plants would be ideal; however this has not routinely been investigated. Most studies have focused on the adaption to supplementation of tannic extracts and not on the short-term effects when feeding the actual plant.

In the present study, the hypothesis that certain phenolic plants, containing different groups of tannins, induce rapid effects to increase phenol content and to partially inhibit RBH and therefore alter the FA composition of bovine milk, was tested. The aim of the study was to quantify the short-term effects of diets containing different tanniferous forages in dairy cows on the presence of phenols in the milk and to perform a detailed analysis of the variations in the milk FA profile.

\section{Material and methods}

\section{Animals, diets and experimental design}

Six late-lactating, Brown Swiss dairy cows were recruited from the research herd of the ETH research station, Chamau (Hünenberg, Switzerland). Cows were in their $1^{\text {st }}-5^{\text {th }}$ lactation with a body weight of $644 \pm 32 \mathrm{~kg}$ and a milk yield of $20.3 \pm 1.4 \mathrm{~kg}$. They were housed separately to allow for individual feed intake measurements and had permanent access to water. Six diets containing different tanniferous forages and lucerne (as the control diet) were fed ad libitum, in a cross-over design, for 3 days each. The experimental protocol was approved by the Cantonal Veterinary Office of Zug (ZG 93/16).

All diets consisted of a mixed basal ration (MBR) ( $(\mathrm{g} / \mathrm{kg}$ dry matter $(\mathrm{DM})$ : grass silage (mixed sward, ryegrass dominated) 495 , maize silage 267 , hay (mixed sward, ryegrass dominated) 91, soyabean meal 87, sugar beet pulp 48, mineral supplement 9 , and feed-grade urea 3 ) and pellets, in a ratio of $0.6: 0.4$. The pellets $(4.5 \mathrm{~mm})$ were produced exclusively for this experiment with our own equipment (Kahl 40P pellet press at $<60{ }^{\circ} \mathrm{C}$, Amandus Kahl $\mathrm{GmbH}$, Reinbek, Germany, aided by steam with Installation Bühler AG, Uzwil, Switzerland). Pellets contained lucerne only $(980 \mathrm{~g} / \mathrm{kg}$ DM lucerne, $20 \mathrm{~g} / \mathrm{kg}$ DM molasses) (control diet) or lucerne combined with the leaves of one of the following plants 
(AlfredGalke,Gittelde, Germany):woodavens(Geum urbanum), rosebay willow (Epilobium angustifolium), blackcurrant (Ribes nigrum), green grape vine (Vitis vinifera), silver birch (Betula pendula) and hazel (Corylus avellana). Plant material was milled to 3-mm particles with a Sigma 5.2 hammer mill (Kuhn AG, Bottighofen, Switzerland) and combined with molasses $(20 \mathrm{~g} / \mathrm{kg} \mathrm{DM})$ and lucerne with a Speedmix DFML-1000 (Bühler AG, Uzwil, Switzerland) before pelleting in proportions intended to reach a total extractable phenol (TEP) content of $60 \mathrm{~g} / \mathrm{kg}$ dietary DM in all tanniferous plant pellets (Terranova et al., 2020). Additionally, 100 g/day of a mineral-vitamin mix (KRONI; Kroni Locher, Altstätten, Switzerland) and $50 \mathrm{~g}$ salt $(390 \mathrm{~g} \mathrm{NaCl} / \mathrm{kg}$, Agrisal, Matile GmbH, Rubigen, Switzerland) were provided.

Each cow received all 6 tanniferous-forage diets and the lucerne diet in a different, randomised order. Pellets were provided separately from the MBR three times daily. Lucerne pellets were fed to all cows for the first 5 days (adaptation period), followed by an additional 3 days of feeding the lucerne-only diet (control). Subsequently, each of the 6 tanniferous-forage diets was fed for 3 days in a randomised order. No 'wash-out' period was applied before the next diet was fed. Milk samples were taken at morning and evening milking on day 3 of feeding each diet.

\section{Data recording and sampling}

Intake and refusals of MBR and plant pellets were measured and recorded daily using calibrated scales (Westfalia Separator). All feed components were sampled, dried $\left(60{ }^{\circ} \mathrm{C}\right.$ for $\left.48 \mathrm{~h}\right)$ and milled $(1 \mathrm{~mm})$ with a centrifugal mill (Retsch, Haan, Germany) before analysis. Cows were milked daily at 05:30 and 16:30 and the corresponding milk yield was recorded. Two sets of milk samples were collected on day 3 in the proportions corresponding to the amounts of morning and evening milk. One set was frozen at $-20{ }^{\circ} \mathrm{C}$ while the other set was conserved with Bronopol ${ }^{\circledR}$ and analysed for fat, protein, lactose and urea content with a MilkoScan FT6000 (Foss, Hillerød, Denmark).

\section{Laboratory analyses of feed samples}

Ether extract (EE) was determined using a Soxhlet extractor (Extraction System B-811, Büchi, Flawil, Switzerland). Phenols, including TEP, condensed tannins (CT) and non-tannin phenols were extracted (Makkar, 2003) with modifications described by Jayanegara et al. (2011) and analysed using a double beam spectrometer (UV-6300, VWR International,
Radnor, PA, USA). The TEP and non-tannin phenol contents were expressed as gallic acid equivalents, while total tannins were assumed as the difference between these. The CT were expressed as leucocyanidin equivalents and hydrolysable tannins (HT) were given as the difference between total tannins and CT. Fatty acids were extracted using a solvent extractor (ASE 200, Dionex, Sunnyvale, CA, USA) using a hexane:propane-2-ol mixture $(3: 2 \mathrm{v} / \mathrm{v})$ and then transformed to FA methyl esters (FAME) (method 2.301; IUPAC, 1987). Cleaning was performed following Wettstein et al. (2001). For FAME analysis, a gas chromatograph (model HP 6890 equipped with a FID detector, Hewlett Packard, Palo Alto, CA, USA) equipped with a CP7421 column $(200 \mathrm{~m} \times 0.25 \mathrm{~mm}, 0.25 \mu \mathrm{m}$; Varian, Darmstadt, Germany) was used. Split injection (1:5) was applied. Internal and external standards used were C11:0 (Fluka, Steinheim, Germany) and commercially available sunflower oil (response factor). The initial temperature was $170{ }^{\circ} \mathrm{C}$, held for $1 \mathrm{~h}$, increased by $5{ }^{\circ} \mathrm{C} / \mathrm{min}$ to $230^{\circ} \mathrm{C}$, held for $32 \mathrm{~min}$, increased by $5{ }^{\circ} \mathrm{C} / \mathrm{min}$ to $250{ }^{\circ} \mathrm{C}$ and held for $15 \mathrm{~min}$. The FA peaks were identified on the basis of retention times of a Supelco 37 Component standard ( $\mathrm{Su}-$ pelco, Bellefonte, PA, USA).

\section{Laboratory analyses of milk samples}

Bronopol ${ }^{\circledR}$ conserved milk samples were analysed for fat and protein contents with a Fourier-transform infrared spectroscopy (MilkoScan FT6000, Foss, Hillerød, Denmark). Total phenols were extracted from milk according to Vázquez et al. (2015) using the Folin-Ciocalteu method and analysed using a double beam spectrometer (UV-6300, VWR International, Radnor, PA, USA). For individual milk FA analysis, samples were defrosted overnight in the fridge $\left(4^{\circ} \mathrm{C}\right) ; 0.5 \mu \mathrm{l}$ of milk was mixed with $5 \mathrm{ml}$ of internal standard (n-heptane containing triundecanoin, tetradecenoic methylate and trivaleranoin). Sodium methylate was used for cold trans-esterification to FAME (Suter et al., 1997). Response factors were derived from triglyceride standards (6:0, 13:0 and 19:0). The same gas chromatograph and column described above were used. The FAME were injected at $1.0 \mu 1$ in a split ratio of $1: 1$ and $1.71 \mathrm{H}_{2} / \mathrm{min}$. The initial temperature $\left(60^{\circ} \mathrm{C}\right.$ ) was held for $12 \mathrm{~min}$, increased by $5^{\circ} \mathrm{C} / \mathrm{min}$ to $170{ }^{\circ} \mathrm{C}$, held for $60 \mathrm{~min}$, increased by $5^{\circ} \mathrm{C} / \mathrm{min}$ to $250^{\circ} \mathrm{C}$ and held for $20 \mathrm{~min}$. FAME identification was performed using the same Supelco 37 Component standard (Supelco, Bellefonte, PA, USA). Fatty acid peak identification was confirmed using Collomb and Bühler (2000) chromatograms. 


\section{Statistical analyses}

Data were analysed with SAS version 9.4 (SAS Institute, Cary, NC, USA). The MIXED procedure was applied using two models. The first model analysing the differences over all treatments including the lucerne-only control diet was:

$$
\mathrm{Y}_{\mathrm{ijkl}}=\mu+\mathrm{D}_{\mathrm{i}}+\mathrm{DIM}_{\mathrm{j}}+\mathrm{c}_{\mathrm{k}}+\mathrm{n}_{\mathrm{l}}+\varepsilon_{\mathrm{ijkl}}
$$

where: $Y_{\mathrm{ijkl}}-$ dependent variable, $\mu$ - overall mean, and $D_{i}$ and DIM - fixed effects of the diet and days in milk, respectively. The cow $c_{k}$ and lactation number $n_{1}$ were set as random factors and $\varepsilon_{\mathrm{ijkl}}$ as the unknown random error.

The SEM and $P$-values shown in Tables 2-5 are given for the first model. In the second model, a pairwise comparison was made for every single treatment compared to the lucerne-only control diet and was:

$$
\mathrm{t}_{\mathrm{ij}}=\left(\overline{\mathrm{y}}_{\mathrm{i}}-\overline{\mathrm{y}}_{\mathrm{j}}\right) / \delta_{\mathrm{ij}}
$$

where: $t_{i}$ and $j_{j}-$ indices of two groups, $\bar{y}_{i}$ and $\overline{\mathrm{Y}}_{\mathrm{j}}$ - LS-means for groups $\mathrm{i}_{\mathrm{i}}$ and $\mathrm{j}, \delta_{\mathrm{ij}}-$ square root of the estimated variance of $\overline{\mathrm{Y}}_{\mathrm{i}}-\overline{\mathrm{Y}}_{\mathrm{j}}$.

Significantly different means from tanniferous treatments to control were marked with an asterix in all tables. The Tukey-Kramer adjustment was applied for multiple comparisons among means. Results are given as least square means with standard errors of the mean. $P<0.05$ was considered significant.

\section{Results}

Wood avens, rosebay willow and green grape vine were characterised by HT, and blackcurrant, hazel and silver birch by CT (Table 1). Lucerne was particularly low in both but contained more HT than CT. All test-plants contained relatively low EE levels (17.3 to $65.6 \mathrm{~g} / \mathrm{kg} \mathrm{DM}$ ) and the same FA (ALA, LA, C16:0 and C18:1 n-9) were the most prevalent in all plants.

When receiving lucerne-only, hazel, blackcurrant, rosebay willow, wood avens, grapevine and silver birch pellets, cows consumed 17.3, 18.0, 15.2, $17.5,17.8,17.5$ and $14.3 \mathrm{~kg} \mathrm{DM} /$ day (data taken from Terranova et al. (2020). The corresponding data on milk yield were 19.2, 17.1, 17.1, 17.7, 18.5, 17.7 and $16.1 \mathrm{~kg} /$ day, those on milk fat content were $47.1,44.7,47.3,45.0,47.1,48.2$ and $47.6 \mathrm{~g} / \mathrm{kg}$, and those on milk protein content were $40.7,40.6,39.8$, $39.7,40.0,40.2$ and $40.5 \mathrm{~g} / \mathrm{kg}$. Data on additional variables are given in Terranova et al. (2020). Rosebay willow induced the highest dietary TEP intake (Table 2) and silver birch the lowest, besides the control (lucerne). In all diets, ALA was the most widely consumed FA, followed by LA, C16:0 and C18:1
Table 1. Analysed mean composition of experimental plants $(n=2)$

\begin{tabular}{llll}
\hline $\begin{array}{l}\text { Diet } \\
\text { components diet Lucerne Hazel }\end{array}$ & $\begin{array}{l}\text { Silver Black- Grape- Wood } \\
\text { birch currant vine avens }\end{array}$ \\
& $\begin{array}{l}\text { Ray } \\
\text { willow }\end{array}$ \\
\hline
\end{tabular}

*Phenols, g/kg dry matter

$\begin{array}{lllllllll}\text { TEP } & 18.5 & 13.4 & 47.8 & 62.4 & 69.8 & 69.0 & 69.3 & 95.1\end{array}$

$\begin{array}{lllllllll}\text { NTP } & 15.5 & 11.7 & 22.0 & 26.0 & 28.2 & 17.5 & 14.3 & 15.9\end{array}$

$\begin{array}{lllllllll}\text { TT } & 3.0 & 1.7 & 25.8 & 36.4 & 41.7 & 51.5 & 55.0 & 79.2\end{array}$

$\begin{array}{lllllllll}\text { CT } & 0.2 & 0.1 & 20.0 & 27.7 & 35.8 & 24.9 & 6.2 & 1.9\end{array}$

$\begin{array}{lllllllll}\text { HT } & 2.9 & 1.5 & 5.8 & 8.6 & 5.8 & 26.6 & 48.9 & 77.3\end{array}$

Ether extract, g/kg dry matter

$\begin{array}{llllllll}29.1 & 17.3 & 23.7 & 65.6 & 36.3 & 36.4 & 23.7 & 24.3\end{array}$

Fatty acids, g/100 g total fatty acids

$\begin{array}{lllllllll}\text { C8:0 } & 0.01 & 0.04 & 0.05 & 0.25 & 0.09 & 0.07 & 0.03 & 0.06\end{array}$

$\begin{array}{lllllllll}\mathrm{C} 10: 0 & 0.02 & 0.26 & 0.13 & 0.12 & 0.21 & 0.13 & 0.10 & 0.11\end{array}$

$\begin{array}{lllllllll}\text { C12:0 } & 0.17 & 0.43 & 0.44 & 0.66 & 1.10 & 0.46 & 0.27 & 0.31\end{array}$

$\begin{array}{lllllllll}\mathrm{C} 14: 0 & 0.30 & 1.38 & 1.38 & 3.23 & 3.67 & 1.44 & 0.68 & 2.16\end{array}$

$\begin{array}{lllllllll}\text { C14:1 } & 0.02 & 0.12 & 0.08 & 0.13 & 0.09 & 0.15 & 0.05 & 0.08\end{array}$

$\begin{array}{lllllllll}C 15: 0 & 0.12 & 0.69 & 0.37 & 0.21 & 0.46 & 0.33 & 0.31 & 0.34\end{array}$

$\begin{array}{lllllllll}\text { C16:0 } & 16.3 & 21.2 & 27.0 & 47.0 & 28.5 & 30.0 & 19.7 & 26.8\end{array}$

$\begin{array}{lllllllll}\text { iso } \mathrm{C} 16: 0 & 0.04 & 0.09 & 0.09 & 0.28 & 0.19 & 0.27 & 0.10 & 0.15\end{array}$

$\begin{array}{lllllllll}\text { anteiso } & 0.03 & 0.13 & 0.10 & 0.24 & 0.07 & 0.16 & 0.17 & 0.08\end{array}$

C16:0

$\begin{array}{lllllllll}\mathrm{C} 17: 0 & 0.15 & 0.38 & 0.42 & 0.82 & 0.51 & 0.49 & 0.35 & 0.43\end{array}$

$\begin{array}{lllllllll}\mathrm{C} 18: 0 & 1.79 & 3.13 & 5.52 & 5.97 & 2.28 & 4.83 & 4.66 & 4.12\end{array}$

$\begin{array}{lllllllll}\mathrm{C} 20: 0 & 0.45 & 1.03 & 0.96 & 5.19 & 0.90 & 1.45 & 1.45 & 2.13\end{array}$

$\begin{array}{lllllllll}\text { C21:0 } & 0.06 & 0.16 & 0.11 & 0.41 & 0.08 & 0.17 & 0.10 & 0.09\end{array}$

$\begin{array}{lllllllll}\mathrm{C} 22: 0 & 0.46 & 1.20 & 1.06 & 4.14 & 0.82 & 1.62 & 1.08 & 0.99\end{array}$

$\begin{array}{lllllllll}\mathrm{C} 23: 0 & 0.06 & 0.05 & 0.09 & 0.39 & 0.14 & 0.34 & 0.12 & 0.28\end{array}$

$\begin{array}{lllllllll}\mathrm{C} 24: 0 & 0.50 & 1.40 & 1.18 & 3.81 & 1.61 & 1.62 & 0.83 & 1.35\end{array}$

$\begin{array}{llllllllll}\text { C12:1 } & 0.15 & 0.21 & 0.26 & 0.18 & 0.42 & 0.32 & 0.20 & 0.27\end{array}$

$\begin{array}{lllllllll}\text { C15:1 } & 0.14 & 0.23 & 0.20 & 1.06 & 0.13 & 0.31 & 0.21 & 0.24\end{array}$

$\begin{array}{lllllllll}\mathrm{C} 16: 1 & 0.20 & 0.73 & 0.90 & 0.94 & 0.35 & 0.52 & 0.39 & 0.47\end{array}$

$\begin{array}{lllllllll}C 17: 1 & 0.03 & 0.05 & 0.06 & 0.21 & 0.09 & 0.30 & 0.12 & 0.08\end{array}$

$\begin{array}{lllllllll}C 18: 1 n-9 & 11.6 & 3.6 & 16.1 & 9.1 & 5.6 & 9.3 & 19.2 & 11.6\end{array}$

$\begin{array}{lllllllll}\mathrm{C} 18: 1 n-11 & 0.52 & 0.75 & 1.13 & 1.02 & 0.78 & 0.77 & 1.11 & 1.03\end{array}$

$\begin{array}{lllllllll}\mathrm{C} 20: 1 n-7 & 0.16 & 0.30 & 0.36 & 0.44 & 0.23 & 0.22 & 0.39 & 0.22\end{array}$

$\begin{array}{llllllllll}\mathrm{C} 20: 1 n-9 & 0.10 & 0.07 & 0.28 & 1.53 & 0.22 & 0.35 & 0.10 & 0.32\end{array}$

$\begin{array}{llllllllll}\mathrm{C} 22: 1 & 0.03 & 0.07 & 0.06 & 0.16 & 0.08 & 0.18 & 0.06 & 0.07\end{array}$

$\begin{array}{lllllllll}\mathrm{C} 24: 1 n-9 & 0.05 & 0.05 & 0.05 & 0.26 & 0.06 & 0.16 & 0.08 & 0.10\end{array}$

$\begin{array}{lllllllll}\text { C18:2 } n-6 & 31.9 & 21.1 & 21.6 & 5.0 & 14.2 & 17.9 & 24.9 & 21.3\end{array}$

$\begin{array}{lllllllll}\mathrm{C} 20: 2 & 0.05 & 0.15 & 0.14 & 0.16 & 0.14 & 0.19 & 0.11 & 0.26\end{array}$

$\begin{array}{lllllllll}\mathrm{C} 22: 2 & 0.14 & 0.62 & 0.31 & 0.83 & 0.68 & 0.61 & 0.30 & 0.34\end{array}$

$\begin{array}{lllllllll}\text { C18:3n-3 } & 34.2 & 39.8 & 19.2 & 5.8 & 35.9 & 24.8 & 22.5 & 24.0\end{array}$

$\begin{array}{lllllllll}\text { C18:3n-6 } & 0.20 & 0.26 & 0.14 & 0.15 & 0.24 & 0.21 & 0.18 & 0.16\end{array}$

$\begin{array}{lllllllll}\mathrm{C} 20: 3 n-3 & 0.05 & 0.14 & 0.08 & 0.15 & 0.10 & 0.17 & 0.07 & 0.08\end{array}$

$\begin{array}{lllllllll}\mathrm{C} 20: 5 n-3 & 0.02 & 0.13 & 0.10 & 0.27 & 0.11 & 0.17 & 0.11 & 0.10\end{array}$

$\begin{array}{lllllllll}\text { SFA } & 20.5 & 31.6 & 38.9 & 72.7 & 40.6 & 43.4 & 29.9 & 39.4\end{array}$

$\begin{array}{lllllllll}\text { MUFA } & 13.0 & 6.2 & 19.5 & 15.0 & 8.1 & 12.6 & 21.9 & 14.5\end{array}$

\begin{tabular}{lllllllll} 
PUFA & 66.5 & 62.2 & 41.6 & 12.3 & 51.3 & 44.0 & 48.2 & 46.2 \\
\hline
\end{tabular}

TEP - total extractable phenols; NTP - non-tannin phenols; TT - total tannins; CT - condensed tannins; HT - hydrolysable tannins; SFA - saturated fatty acid; MUFA - mono-unsaturated fatty acid; PUFA - polyunsaturated fatty acid; " values sourced or calculated (pooled mean of two batches) from Terranova et al. (2020) 
Table 2. Intake of total extractable phenols, ether extract and fatty acids ( $\mathrm{g} /$ day) ( 3 days of feeding dairy cows experimental diets), $n=6$ per diet

\begin{tabular}{|c|c|c|c|c|c|c|c|c|c|}
\hline \multirow[b]{2}{*}{ Indices } & \multicolumn{7}{|c|}{ Basal diet supplemented with } & \multirow[b]{2}{*}{ SEM } & \multirow[b]{2}{*}{$P$-value } \\
\hline & lucerne & hazel & $\begin{array}{l}\text { silver } \\
\text { birch }\end{array}$ & $\begin{array}{l}\text { black- } \\
\text { currant }\end{array}$ & $\begin{array}{l}\text { grape } \\
\text { vine }\end{array}$ & $\begin{array}{l}\text { wood } \\
\text { avens }\end{array}$ & $\begin{array}{l}\text { rosebay } \\
\text { willow }\end{array}$ & & \\
\hline $\begin{array}{l}\text { Total extractable } \\
\text { phenols }\end{array}$ & 289 & $545^{b^{*}}$ & $460^{b^{*}}$ & $526^{b^{*}}$ & $640^{\mathrm{ab}^{*}}$ & $642^{\mathrm{ab}^{*}}$ & $816^{a^{*}}$ & 43.4 & $<0.001$ \\
\hline Ether extract & 275 & $317^{\mathrm{a}^{*}}$ & $252^{b}$ & $265^{b}$ & $267^{b}$ & $320^{a^{*}}$ & $285^{a}$ & 7.49 & $<0.001$ \\
\hline \multicolumn{10}{|l|}{ Fatty acids } \\
\hline C8:0 & 0.051 & 0.074 & $0.122^{*}$ & 0.073 & 0.063 & 0.063 & 0.071 & 0.0097 & $<0.001$ \\
\hline $\mathrm{C} 10: 0$ & 0.203 & $0.178^{a}$ & $0.087 \mathrm{c}^{*}$ & 0.152 & $0.113^{\mathrm{bc}^{*}}$ & 0.152 & $0.122^{\mathrm{bc}^{*}}$ & 0.0117 & $<0.001$ \\
\hline $\mathrm{C} 12: 0$ & 0.629 & 0.816 & $0.613^{b}$ & $0.932^{2^{*}}$ & $0.604^{b}$ & $0.641^{b}$ & $0.577^{\mathrm{b}}$ & 0.0571 & $<0.001$ \\
\hline C14:0 & 1.51 & 2.07 & $1.89^{a}$ & $2.56^{\mathrm{a}^{*}}$ & $1.41^{b}$ & $1.36^{b}$ & $2.18^{a}$ & 0.208 & 0.002 \\
\hline C14:1 & 0.120 & 0.126 & 0.095 & 0.095 & 0.127 & 0.102 & 0.101 & 0.0070 & 0.004 \\
\hline C15:0 & 0.691 & $0.636^{a}$ & $0.335^{\mathrm{d}^{*}}$ & $0.495^{\mathrm{bc}}$ & $0.433^{\mathrm{cd}^{\mathrm{d}}}$ & $0.581^{a}$ & $0.499^{\mathrm{bc}}$ & 0.0268 & $<0.001$ \\
\hline C16:0 & 48.2 & $63.0^{a^{*}}$ & $53.0^{\mathrm{a}}$ & $49.7^{b}$ & $51.0^{\mathrm{b}}$ & $55.7^{\mathrm{a}}$ & $54.0^{a b}$ & 2.34 & 0.003 \\
\hline iso C16:0 & 0.148 & 0.188 & 0.198 & 0.189 & $0.236^{*}$ & 0.198 & 0.199 & 0.0143 & 0.014 \\
\hline anteiso C16:0 & 0.138 & $0.157^{b}$ & $0.152^{\mathrm{bc}}$ & $0.094^{c}$ & $0.141^{\mathrm{bc}}$ & $0.240^{\mathrm{a}^{*}}$ & $0.115^{\text {bc }}$ & 0.0125 & $<0.001$ \\
\hline C17:0 & 0.571 & $0.764^{*}$ & 0.647 & 0.595 & 0.593 & 0.702 & 0.636 & 0.0413 & 0.029 \\
\hline C18:0 & 5.77 & $9.55^{a^{*}}$ & $6.11^{\mathrm{bc}}$ & $5.00^{c}$ & $6.40^{\mathrm{bc}}$ & $8.76^{a^{*}}$ & $6.76^{b}$ & 0.328 & $<0.001$ \\
\hline C20:0 & 1.60 & $1.96^{b}$ & $2.97^{a^{*}}$ & $1.42^{\mathrm{c}}$ & $1.73^{\mathrm{b}}$ & $2.50^{a b^{*}}$ & $2.48^{a}$ & 0.200 & $<0.001$ \\
\hline C21:0 & 0.222 & $0.240^{\mathrm{ab}}$ & $0.284^{a}$ & $0.164^{c}$ & $0.215^{\mathrm{abc}}$ & $0.235^{\mathrm{abc}}$ & $0.187^{b c}$ & 0.0159 & $<0.001$ \\
\hline $\mathrm{C} 22: 0$ & 1.74 & $2.08^{a}$ & $2.59^{a^{*}}$ & $1.41^{b}$ & $1.86^{\mathrm{ab}}$ & $2.13^{a}$ & $1.70^{\mathrm{b}}$ & 0.160 & $<0.001$ \\
\hline C23:0 & 0.161 & $0.228^{b}$ & $0.280^{\mathrm{ab}^{*}}$ & $0.204^{b}$ & $0.313^{\mathrm{a}^{*}}$ & $0.256^{\mathrm{ab}^{*}}$ & $0.329^{\mathrm{a}^{*}}$ & 0.0168 & $<0.001$ \\
\hline $\mathrm{C} 24: 0$ & 1.95 & 2.30 & 2.55 & 1.91 & 1.94 & 1.96 & 2.04 & 0.1600 & 0.065 \\
\hline C12:1 & 0.446 & $0.579^{a^{*}}$ & $0.381^{b}$ & $0.530^{\mathrm{a}}$ & $0.483^{a b}$ & $0.523^{a}$ & $0.507^{a}$ & 0.0220 & $<0.001$ \\
\hline C15:1 & 0.435 & $0.500^{b}$ & $0.698^{a^{*}}$ & $0.357^{b}$ & $0.453^{b}$ & $0.516^{a b}$ & $0.459^{b}$ & 0.0390 & $<0.001$ \\
\hline C16:1 & 0.886 & $1.366^{a^{*}}$ & $0.793^{b}$ & $0.612^{\mathrm{c}^{*}}$ & $0.709^{b}$ & $0.845^{b}$ & $0.762^{b}$ & 0.0423 & $<0.001$ \\
\hline C17:1 & 0.090 & $0.124^{c}$ & $0.142^{\mathrm{bc} c^{*}}$ & $0.107^{c}$ & $0.217^{a^{*}}$ & $0.187^{a^{*}}$ & $0.118^{c}$ & 0.0111 & $<0.001$ \\
\hline C18:1 n-9 & 26.9 & $41.3^{a^{*}}$ & $28.1^{c}$ & $27.6^{c}$ & $29.6^{\text {bc }}$ & $45.1^{2^{*}}$ & $32.9^{b^{*}}$ & 0.99 & $<0.001$ \\
\hline C18:1 n-11 & 1.59 & $2.29^{\mathrm{a}^{*}}$ & $1.51^{\mathrm{c}}$ & $1.52^{\mathrm{bc}}$ & $1.53^{\mathrm{bc}}$ & $2.29^{a^{*}}$ & $1.86^{b}$ & 0.073 & $<0.001$ \\
\hline C20:1 n-7 & 0.535 & $0.723^{\mathrm{a}^{*}}$ & $0.518^{b}$ & $0.469^{b}$ & $0.468^{b}$ & $0.763^{\mathrm{a}^{*}}$ & $0.506^{b}$ & 0.0265 & $<0.001$ \\
\hline $\mathrm{C} 20: 1 n-9$ & 0.251 & $0.491^{b}$ & $0.799^{a^{*}}$ & $0.319^{b}$ & $0.390^{b}$ & $0.307^{b}$ & $0.435^{b}$ & 0.0545 & $<0.001$ \\
\hline C22:1 & 0.104 & $0.126^{a b}$ & $0.122^{b}$ & $0.101^{b}$ & $0.160^{\mathrm{a}^{*}}$ & $0.126^{a b}$ & $0.114^{b}$ & 0.0075 & $<0.001$ \\
\hline $\mathrm{C} 24: 1 n-9$ & 0.145 & $0.167^{a b}$ & $0.212^{a^{*}}$ & $0.142^{b}$ & $0.195^{a^{*}}$ & $0.193^{a^{*}}$ & $0.184^{a b}$ & 0.0105 & $<0.001$ \\
\hline C18:2 n-6 (LA) & 81.3 & $90.5^{2^{*}}$ & $69.9^{\mathrm{d}^{*}}$ & $75.4^{\text {cd }}$ & $77.6^{\mathrm{bc}}$ & $94.5^{\mathrm{a}^{*}}$ & $83.2^{b}$ & 1.32 & $<0.001$ \\
\hline $\mathrm{C} 20: 2$ & 0.194 & $0.244^{a^{b^{*}}}$ & $0.163^{d}$ & $0.174^{\text {cd }}$ & $0.201^{\mathrm{bcd}}$ & $0.217^{\mathrm{bc}}$ & $0.282^{\mathrm{a}^{*}}$ & 0.0106 & $<0.001$ \\
\hline $\mathrm{C} 22: 2$ & 0.681 & 0.611 & 0.613 & 0.647 & 0.622 & 0.612 & 0.535 & 0.0460 & 0.493 \\
\hline C18:3 n-3 (ALA) & 97.9 & $92.8^{\mathrm{ab}}$ & $75.0^{\mathrm{c}^{*}}$ & $91.5^{\mathrm{ab}}$ & $86.1^{\mathrm{b}^{*}}$ & $96.7^{\mathrm{a}}$ & $90.0^{\mathrm{ab}}$ & 1.93 & $<0.001$ \\
\hline C18:3n-6 & 0.579 & $0.563^{a b}$ & $0.475^{c^{*}}$ & $0.542^{\mathrm{abc}}$ & $0.530^{\mathrm{bc}}$ & $0.607^{a}$ & $0.533^{\text {bc }}$ & 0.0147 & $<0.001$ \\
\hline $\mathrm{C} 20: 3 n-3$ & 0.201 & $0.195^{\mathrm{ab}}$ & $0.172^{\mathrm{ab}}$ & $0.167^{b}$ & $0.205^{\mathrm{a}}$ & $0.188^{a b}$ & $0.174^{\mathrm{ab}}$ & 0.0080 & 0.007 \\
\hline C20:5 n-3 & 0.130 & $0.154^{\mathrm{ab}}$ & $0.156^{\mathrm{ab}}$ & $0.106^{b}$ & $0.144^{\mathrm{ab}}$ & $0.169^{a}$ & $0.126^{\mathrm{ab}}$ & 0.0122 & 0.018 \\
\hline
\end{tabular}

a-d - means within rows with different superscripts are significantly different at $P<0.05 ;{ }^{*}-$ means are different from those of lucerne at $P<0.05$; SEM - standard error of the mean

$n-9$. Test-plants significantly increased milk phenol contents when compared to the control (Table 3). Wood avens resulted in a significantly higher milk TP excretion in comparison to lucerne (Table 4). Feeding different plant-pellets significantly affected the proportions of certain individual $\mathrm{FA}$ in the milk fat including C15.0, C15:1, C16:1, iso $\mathrm{C} 17$ and the RBH intermediates RA, C18:2 cis-9 cis-11, C18:2 trans-9 trans-11 (Table 3 ) as well as the excretion of RA
(Table 4). Transfer rates for TP from feed to milk were significantly lower in the test-plants in comparison to lucerne (Table 5). Transfer of FA from feed to milk was on average $116 \mathrm{~g} / \mathrm{kg}$ for LA and $67 \mathrm{~g} / \mathrm{kg}$ for ALA for all diets. Significant differences between hazel, blackcurrant, rosebay willow, grape vine and the control were observed when comparing the intake of these two FA with the excretion of RA. Numerically the same was true for VA. 
Table 3. Dietary effects on total phenol content in milk ( $\mathrm{mg} / \mathrm{l})$ and on individual fatty acid proportions ( $\mathrm{g} / 100 \mathrm{~g}$ total fatty acids) in milk fat ( 3 days of feeding dairy cows experimental diets), $n=6$ per diet

\begin{tabular}{|c|c|c|c|c|c|c|c|c|c|}
\hline \multirow[b]{2}{*}{ Indices } & \multicolumn{7}{|c|}{ Basal diet supplemented with } & \multirow[b]{2}{*}{ SEM } & \multirow[b]{2}{*}{$P$-value } \\
\hline & lucerne & hazel & $\begin{array}{l}\text { silver } \\
\text { birch }\end{array}$ & $\begin{array}{l}\text { black- } \\
\text { currant }\end{array}$ & $\begin{array}{l}\text { grape } \\
\text { vine }\end{array}$ & $\begin{array}{l}\text { wood } \\
\text { avens }\end{array}$ & $\begin{array}{l}\text { rosebay } \\
\text { willow }\end{array}$ & & \\
\hline Total phenols & 9.7 & 12.3 & 9.8 & 11.7 & 11.4 & 13.0 & 11.7 & 0.75 & 0.035 \\
\hline \multicolumn{10}{|l|}{ Fatty acids (FA) } \\
\hline $\mathrm{C} 4: 0$ & 1.30 & 1.20 & 1.23 & 1.20 & 1.25 & 1.21 & 1.21 & 0.08 & 0.96 \\
\hline C6:0 & 1.43 & 1.41 & 1.49 & 1.38 & 1.47 & 1.48 & 1.46 & 0.04 & 0.46 \\
\hline C8:0 & 0.04 & 0.05 & 0.04 & 0.05 & 0.04 & 0.05 & 0.05 & 0.002 & 0.65 \\
\hline C10:0 & 3.27 & 3.30 & 3.56 & 3.02 & 3.26 & 3.44 & 3.41 & 0.12 & 0.11 \\
\hline C10:1 & 0.41 & 0.42 & 0.43 & 0.36 & 0.40 & 0.42 & 0.39 & 0.02 & 0.36 \\
\hline C12:0 & 4.40 & 4.59 & 4.96 & 4.05 & 4.37 & 4.64 & 4.61 & 0.20 & 0.11 \\
\hline iso $\mathrm{C} 12: 0$ & 0.15 & 0.14 & 0.15 & 0.12 & 0.13 & 0.14 & 0.13 & 0.01 & 0.27 \\
\hline C13:0 & 0.13 & $0.13^{a b}$ & $0.15^{\mathrm{a}}$ & $0.11^{\mathrm{b}}$ & $0.11^{\mathrm{b}}$ & $0.12^{b}$ & $0.12^{\mathrm{b}}$ & 0.006 & 0.003 \\
\hline iso $\mathrm{C} 13: 0$ & 0.17 & 0.14 & 0.14 & 0.16 & 0.16 & 0.14 & 0.16 & 0.009 & 0.11 \\
\hline C14:0 & 14.7 & 15.3 & 15.2 & 14.5 & 15.2 & 15.4 & 15.4 & 0.28 & 0.14 \\
\hline anteiso C14:0 & 0.67 & $0.54^{*}$ & $0.54^{*}$ & $0.55^{*}$ & $0.56^{*}$ & $0.55^{*}$ & $0.55^{*}$ & 0.02 & $<0.001$ \\
\hline C15:0 & 1.59 & $1.38^{*}$ & 1.44 & $1.31^{*}$ & $1.30^{*}$ & $1.32^{*}$ & $1.33^{*}$ & 0.03 & $<0.001$ \\
\hline C16:0 & 32.9 & $38.1^{*}$ & 36.8 & 34.6 & 36.1 & 35.1 & 35.0 & 0.92 & 0.01 \\
\hline anteiso $\mathrm{C} 16: 0$ & 0.04 & 0.04 & 0.05 & 0.04 & 0.04 & 0.04 & 0.04 & 0.001 & 0.05 \\
\hline C17:0 & 0.64 & 0.55 & 0.59 & 0.62 & 0.59 & 0.56 & 0.59 & 0.03 & 0.28 \\
\hline iso $\mathrm{C} 17: 0$ & 0.10 & $0.06^{*}$ & $0.06^{*}$ & $0.07^{*}$ & $0.07^{*}$ & $0.06^{*}$ & $0.06^{*}$ & 0.01 & $<0.001$ \\
\hline C18:0 & 6.91 & 5.74 & 6.20 & 7.07 & 6.59 & 6.67 & 7.10 & 0.37 & 0.14 \\
\hline C20:0 & 0.10 & $0.08^{b}$ & $0.10^{\mathrm{ab}}$ & $0.09^{a b}$ & $0.10^{\mathrm{ab}}$ & $0.10^{\mathrm{ab}}$ & $0.11^{\mathrm{a}}$ & 0.01 & 0.08 \\
\hline C12:1 & 0.15 & 0.15 & 0.16 & 0.12 & 0.13 & 0.12 & 0.14 & 0.02 & 0.41 \\
\hline C14:1 & 1.58 & 1.66 & 1.59 & 1.53 & 1.61 & 1.69 & 1.51 & 0.10 & 0.89 \\
\hline C15:1 & 0.39 & $0.28^{*}$ & $0.29^{*}$ & $0.33^{*}$ & $0.30^{*}$ & $0.33^{*}$ & $0.32^{*}$ & 0.01 & $<0.001$ \\
\hline C16:1 & 0.39 & $0.31^{*}$ & $0.31^{*}$ & $0.32^{*}$ & $0.32^{*}$ & $0.31^{*}$ & $0.31^{*}$ & 0.01 & $<0.001$ \\
\hline C16:1 n-7 & 1.60 & 1.77 & 1.70 & 1.72 & 1.66 & 1.64 & 1.49 & 0.11 & 0.62 \\
\hline C17:1 & 0.29 & 0.25 & 0.27 & 0.29 & 0.26 & 0.25 & 0.24 & 0.01 & 0.10 \\
\hline C18:1 n-9 & 16.8 & 15.5 & 15.9 & 18.6 & 16.5 & 16.7 & 16.8 & 1.06 & 0.55 \\
\hline cis-10 C18:1 & 0.30 & $0.28^{\mathrm{ab}}$ & $0.24^{\mathrm{b}^{*}}$ & $0.28^{\mathrm{ab}}$ & $0.29^{\mathrm{ab}}$ & $0.30^{\mathrm{a}}$ & $0.31^{\mathrm{a}}$ & 0.01 & 0.004 \\
\hline cis-11 C18:1 & 0.35 & 0.38 & 0.39 & $0.45^{*}$ & 0.40 & 0.38 & 0.41 & 0.02 & 0.06 \\
\hline cis-12 C18:1 & 0.21 & 0.22 & 0.19 & 0.21 & 0.21 & 0.22 & 0.22 & 0.01 & 0.49 \\
\hline cis-13 C18:1 & 0.08 & 0.07 & 0.07 & 0.08 & 0.08 & 0.07 & 0.06 & 0.01 & 0.51 \\
\hline cis-14 + 18:1 trans-16 C18:1 & 0.09 & 0.09 & 0.07 & 0.08 & 0.09 & 0.88 & 0.88 & 0.01 & 0.92 \\
\hline trans-6-8 C18:1 & 0.18 & 0.15 & 0.14 & 0.14 & 0.14 & 0.15 & 0.17 & 0.01 & 0.21 \\
\hline trans-9 C18:1 & 0.18 & 0.18 & 0.16 & 0.19 & 0.19 & 0.18 & 0.16 & 0.01 & 0.42 \\
\hline trans-10 C18:1 & 0.27 & 0.19 & 0.17 & 0.27 & 0.19 & 0.22 & 0.20 & 0.04 & 0.63 \\
\hline trans-11 C18:1 (VA) & 2.59 & 0.81 & 0.74 & 1.00 & 0.93 & 0.86 & 0.85 & 0.58 & 0.29 \\
\hline trans-12 C18:1 & 0.22 & 0.26 & 0.18 & 0.22 & 0.23 & 0.24 & 0.21 & 0.02 & 0.19 \\
\hline cis-9 C20:1 & 0.12 & $0.11^{b}$ & $0.11^{a b}$ & $0.11^{b}$ & $0.11^{a b}$ & $0.12^{\mathrm{ab}}$ & $0.12^{\mathrm{a}}$ & 0.003 & $<0.01$ \\
\hline cis-11 C20:1 & 0.05 & $0.02^{*}$ & 0.03 & 0.04 & $0.03^{*}$ & $0.03^{*}$ & $0.03^{*}$ & 0.004 & 0.001 \\
\hline C18:2 n-6 (LA) & 1.38 & 1.32 & 1.35 & 1.46 & 1.43 & 1.48 & 1.52 & 0.04 & 0.02 \\
\hline cis-9, cis-11 C18:2 & 0.05 & $0.03^{*}$ & $0.03^{*}$ & $0.03^{*}$ & $0.03^{*}$ & $0.03^{*}$ & $0.03^{*}$ & 0.002 & $<0.001$ \\
\hline cis- 9 , cis-15 C18:2 & 0.36 & 0.31 & $0.25^{*}$ & 0.33 & 0.30 & 0.30 & 0.31 & 0.02 & $<0.01$ \\
\hline trans- 8 , cis- $9+18: 2$ cis- 12 , trans- 13 C18:2 & 20.26 & 0.20 & 0.16 & 0.19 & 0.19 & 0.16 & 0.16 & 0.02 & 0.12 \\
\hline cis-9, trans-11 C18:2 (RA) & 0.67 & $0.51^{*}$ & $0.46^{*}$ & 0.56 & $0.53^{*}$ & $0.54^{*}$ & $0.49^{*}$ & 0.03 & $<0.001$ \\
\hline cis-9, trans-12 C18:2 & 0.13 & 0.05 & 0.05 & 0.06 & 0.06 & 0.06 & 0.06 & 0.01 & 0.008 \\
\hline trans -9 , trans- $11+18: 2$ cis- 12 , cis- 15 C18:2 & 20.03 & 0.03 & 0.04 & 0.03 & 0.03 & 0.04 & 0.03 & 0.002 & 0.29 \\
\hline trans-9, trans-11 C18:2 & 0.67 & $0.51^{*}$ & $0.46^{*}$ & 0.56 & $0.53^{*}$ & $0.54^{*}$ & $0.49^{*}$ & 0.03 & $<0.001$ \\
\hline C18:3 n-3 (ALA) & 0.91 & $0.83^{\mathrm{ab}}$ & $0.75^{b}$ & $1.00^{\mathrm{a}}$ & $0.89^{\mathrm{ab}}$ & $0.95^{\mathrm{a}}$ & $0.98^{\mathrm{a}}$ & 0.40 & $<0.01$ \\
\hline C18:3n-6 & 0.05 & 0.04 & 0.04 & 0.04 & 0.04 & 0.05 & 0.05 & 0.003 & 0.070 \\
\hline$\sum$ saturated FA & 67.4 & 71.8 & 71.8 & 68.0 & 70.5 & 70.1 & 70.4 & 1.10 & 0.05 \\
\hline$\sum$ monounsaturated FA & 26.3 & 23.1 & 23.2 & 26.4 & 24.1 & 24.4 & 24.1 & 0.94 & 0.09 \\
\hline $\bar{\Sigma}$ polyunsaturated FA & 4.38 & $3.76^{*}$ & $3.62^{*}$ & 4.17 & 3.96 & 4.15 & 4.11 & 0.13 & 0.003 \\
\hline$\sum n-3 F A$ & 1.10 & $0.99^{a b}$ & $0.95^{b}$ & $1.19^{a}$ & $1.08^{\mathrm{ab}}$ & $1.14^{\mathrm{ab}}$ & $1.17^{\mathrm{a}}$ & 0.04 & 0.005 \\
\hline$\sum n-6 \mathrm{FA}$ & 1.77 & $1.62^{\mathrm{b}}$ & $1.67^{\mathrm{ab}}$ & $1.76^{\mathrm{ab}}$ & $1.73^{a b}$ & $1.86^{\mathrm{a}}$ & $1.85^{\mathrm{ab}}$ & 0.05 & 0.022 \\
\hline$\sum n-6 \mathrm{FA} / \sum n-3 \mathrm{FA}$ & 1.61 & $1.64^{\mathrm{ab}}$ & $1.77^{\mathrm{a}}$ & $1.48^{b}$ & $1.61^{\mathrm{ab}}$ & $1.64^{\mathrm{ab}}$ & $1.59^{\mathrm{ab}}$ & 0.04 & 0.006 \\
\hline
\end{tabular}


Table 4. Excretion of total phenols, fat and key fatty acids ${ }^{1}$ in milk ( $g /$ day) ( 3 days of feeding dairy cows experimental diets), $n=6$ per diet

\begin{tabular}{|c|c|c|c|c|c|c|c|c|c|}
\hline \multirow[b]{2}{*}{ Indices } & \multicolumn{7}{|c|}{ Basal diet supplemented with } & \multirow[b]{2}{*}{ SEM } & \multirow[b]{2}{*}{$P$-value } \\
\hline & lucerne & azel & $\begin{array}{l}\text { silver } \\
\text { birch }\end{array}$ & $\begin{array}{l}\text { black- } \\
\text { currant }\end{array}$ & $\begin{array}{l}\text { grape } \\
\text { vine }\end{array}$ & $\begin{array}{l}\text { wood } \\
\text { avens }\end{array}$ & $\begin{array}{l}\text { rosebay } \\
\text { willow }\end{array}$ & & \\
\hline Total phenols & 0.184 & 0.222 & 0.150 & 0.197 & 0.205 & $0.251^{*}$ & 0.202 & 0.0141 & 0.001 \\
\hline Fat & 904 & 764 & 766 & 809 & 853 & 871 & 797 & 1.8 & 0.754 \\
\hline \multicolumn{10}{|l|}{ Fatty acids } \\
\hline C18:2 n-6 (LA) & 9.77 & $8.78^{\mathrm{ab}}$ & $8.29^{b}$ & $9.40^{\mathrm{ab}}$ & $9.56^{\mathrm{ab}}$ & $10.9^{\mathrm{a}}$ & $9.66^{\mathrm{ab}}$ & 0.5554 & 0.055 \\
\hline C18:3 n-3 (ALA) & 6.43 & $5.51^{\mathrm{ab}}$ & $4.61^{b}$ & $6.44^{\mathrm{ab}}$ & $6.00^{\mathrm{ab}}$ & $7.04^{\mathrm{a}}$ & $6.23^{\mathrm{ab}}$ & 0.421 & 0.010 \\
\hline trans-11 C18:1 (VA) & 17.70 & 5.31 & 4.54 & 6.48 & 6.27 & 6.42 & 5.48 & 3.848 & 0.243 \\
\hline cis-9, trans-11 C18:2(RA) & 4.78 & $3.38^{a b^{*}}$ & $2.79^{b *}$ & $3.64^{\mathrm{ab}^{\star}}$ & $3.52^{\mathrm{ab}^{*}}$ & $4.02^{\mathrm{a}}$ & $3.12^{a b^{*}}$ & 0.249 & $<0.001$ \\
\hline
\end{tabular}

${ }^{\text {ab }}$ - means within rows with different superscripts are significantly different at $P<0.05$; ${ }^{*}$ - means are different from those of lucerne at $P<0.05$; SEM - standard error of the mean; ${ }^{1}$ - only fatty acids making up $>0.1 \mathrm{~g} / 100 \mathrm{~g}$ fatty acid methyl esters are displayed, all others were considered as traces

Table 5. Total phenol and key fatty acid excretion in milk in relation to total intake $(\mathrm{g} / \mathrm{kg})$ ( 3 days of feeding dairy cows experimental diets), $\mathrm{n}=6$ per diet

\begin{tabular}{|c|c|c|c|c|c|c|c|c|c|}
\hline \multirow[b]{2}{*}{ Indices } & \multicolumn{7}{|c|}{ Basal diet supplemented with } & \multirow[b]{2}{*}{ SEM } & \multirow[b]{2}{*}{$P$-value } \\
\hline & lucerne & hazel & $\begin{array}{l}\text { silver } \\
\text { birch }\end{array}$ & $\begin{array}{l}\text { black- } \\
\text { currant }\end{array}$ & $\begin{array}{l}\text { grape } \\
\text { vine }\end{array}$ & $\begin{array}{l}\text { wood } \\
\text { avens }\end{array}$ & $\begin{array}{l}\text { rosebay } \\
\text { willow }\end{array}$ & & \\
\hline Total phenols & 0.637 & $0.408^{*}$ & $0.0004^{*}$ & $0.411^{*}$ & $0.313^{*}$ & $0.406^{*}$ & $0.248^{*}$ & 0.0440 & $<0.001$ \\
\hline C18:2 n-6 & 120 & 97 & 119 & 125 & 123 & 115 & 116 & 6.3 & 0.080 \\
\hline C18:3n-3 & 65.7 & 59.3 & 61.4 & 70.1 & 69.8 & 72.9 & 69.2 & 4.32 & 0.274 \\
\hline trans-11 C18:1* & 101 & 29.0 & 31.2 & 39.0 & 38.3 & 33.6 & 31.6 & 22.26 & 0.282 \\
\hline cis-9, trans-11 C18:2** & 26.7 & $18.5^{*}$ & $19.2^{*}$ & 21.8 & 21.5 & 21.1 & $18.0^{*}$ & 1.36 & 0.002 \\
\hline
\end{tabular}

${ }^{*}$ - means are different from those of lucerne at $P<0.05$; ${ }^{* *}$-in relation to intakes of $C 18: 2 n-6$ plus $C 18: 3 n-3 ;$ SEM - standard error of the mean

\section{Discussion}

Phenolic compounds including tannins are of particular interest in ruminant nutrition. A positive or negative outcome is largely determined by dose (Frutos et al., 2004). High doses of tannins have been reported to decrease palatability due to their astringent taste and exert anti-nutritional effects. Astringency is caused by tannins (mainly CT) binding to proteins in the saliva and subsequently aggregating to form insoluble protein-complexes in the mouth. This causes saliva to lose its lubricating ability resulting in a dry mouth feel. Epigallocatechin gallate in tannins can also stimulate, stimulate the trigeminal nerve trigeminal nerve causing a rough mouth feel. These mechanisms may lead to decreased energy intake and subsequent milk yield decline in dairy cows. However, in low to moderate doses they have been shown to decrease bloat, helminth infestations and methane production (Vasta et al., 2019; Frutos et al., 2004). We investigated if the transfer of phenols to milk, and their subsequent effects on RBH, could rapidly improve the composition of ruminant-derived food products.

Phenols. Recent studies have demonstrated that phenols consumed by ruminants are capable of transfer from diet to milk in limited amounts (Alenisan et al., 2017; Leparmarai et al., 2019).
In the present study an increased excretion of phenols in the milk of cows fed wood avens in comparison to lucerne and a trend towards the same with the other tanniferous test-forages, except silver birch were demonstrated. However, the overall excretion of phenols in milk was very low (average of $29 \mathrm{mg} / 100 \mathrm{~g}$ ), therefore no 'phenol-enriched' milk benefits can be claimed from these diets. It should be noted that different methods of extraction are routinely performed to derive phenols from solid (feed) vs. liquid (milk) phases. The TEP (Makkar, 2003) are obtained from feed whereas TP (Vázquez et al., 2015) are extracted from milk introducing a possible element of bias. Nevertheless, our results coincide with those by Soberon et al. (2012) who recorded a very low recovery rate of supplemented powdered ferulic acid (a phenolic lignin precursor found in plant cell walls) of $20 \mathrm{mg} / 100 \mathrm{~g}$ milk. Additionally, Leparmarai et al. (2019) demonstrated ruminant species differences with a weak relationship between phenol intake and excretion in sheep milk but not in goat's milk.

Milk fatty acids. Milk FA are dynamic and derived relatively equally from the diet and the ruminal microbiota of dairy cows. Long chain FA and about half of C16:0 are solicited from the diet and lipolysis of adipose tissue triacylglycerols. Short- and medium-chain FA (C4:0-C14:0) plus the remaining 
C16:0 fraction are formed by de novo synthesis in the mammary gland (Månsson, 2008). Ruminal microbes are responsible for the formation of $\mathrm{C} 15: 0$ and $\mathrm{C} 17: 0$ (Månsson, 2008) as well as the progressive isomerisation and saturation of dietary PUFA to (ultimately) C18:0. (Lourenço et al., 2010; Vasta et al., 2019). In the present study, the onset of effects by phenolic compounds on biochemical pathways of RHB and the subsequent modification of the milk FA composition, were of primary interest.

Popular phenol-based strategies to influence $\mathrm{RBH}$, particularly that of ALA and LA (commonly the most abundant PUFA in dairy cow diets) include the addition of tanniferous feeds (Vasta et al., 2009; Majewska and Kowalik, 2020) or extracts (Khiaosa-Ard et al., 2009); these impose direct or indirect effects (or both) on rumen microbes. When PUFA biohydrogenation is not completed, VA may accumulate and be partially transformed to RA in the mammary gland (Lourenço et al., 2010). Consequently an increase in VA, RA or both in milk fat occurs (Hanuš et al., 2018; Castro et al., 2019). The present study reveals the opposite, RA and VA decreased by 29 and $68 \%$ respectively, suggesting that although microbial activity was inhibited (this is evident by these results and the reduction of $\mathrm{C} 15: 0$ and $\mathrm{C} 17: 0$ ) the bacteria responsible for $\mathrm{RBH}$ were not. Proportions of ALA and LA did not increase in the milk when our 6 tanniferous-forage diets (naturally elevated in CT and HT in comparison to lucerne) were fed for 3 days indicating that PUFA were not protected from biohydrogenation in this limited time period. C18:0 remained stable suggesting a possible indifference to $\mathrm{RBH}$ or that the feeding time was too short to exhibit a physiological effect. Chilliard et al. (2007) reported that ALA and LA are transferred in proportions of 7 and $15 \%$ from the ruminant's diet to the milk, respectively. This corresponds almost exactly with our findings of about 7\% ALA and $12 \%$ LA being transferred to the milk. Therefore, other explanations for the depletion in biohydrogenation intermediates should be explored.

Nevertheless, these 6 tanniferous diets produced significant effects on the proportions of many (often minor) FA in the milk after just 3 days. These findings support those of Denninger et al. (2020) who showed an increase in minor FA after supplementation with Acacia tannins after $17 \mathrm{~h}$ and clear effects within 3 days. This, and the variations found in the odd-chain FA $(15: 0,17: 0)$ proportions, confirm that rumen microbes involved in lipid metabolism seem to adapt rapidly and specifically to tanniferous forages and extracts (Denninger et al, 2020); however, this is not primarily demonstrated in $\mathrm{RBH}$.

\section{Conclusions}

Supplementing dairy cow diets with natural, tanniferous forages induced rapid changes in bovine milk fatty acid (FA) composition and phenol content confirming the stated hypothesis. Certain individual FA including C15-C17, the long-chain FA (specifically $\alpha$-linolenic acid) and ruminal biohydrogenation $(\mathrm{RBH})$ intermediates (rumenic acid and vaccenic acid) were particularly affected. However, 3 days is obviously not long enough to protect significant amounts of polyunsaturated FA from RBH or significantly enhance RBH intermediates. From these results, we are not able to reliably conclude whether condensed or hydrolysable tannins are the main drivers of RBH inhibition and ambiguity remains regarding $\mathrm{RBH}$ effects on milk FA with prolonged feeding periods of these 6 tanniferous forages. Further research should concentrate on longer feeding periods as well as the inclusion of other natural, biodiverse forages. Wood avens resulted in significantly increased amounts of phenols excreted in milk when compared to lucerne and silver birch. However, transfer of phenols to milk was very low with all tanniferous forage diets. This is possibly due to tannins comprising the bulk of phenolic compounds in our 6 test-plants. Therefore, further investigations should focus on the efficient transfer of non-tannic, phenolic compounds from bovine diets to milk.

\section{Acknowledgements}

We would like to thank Carmen Kunz, Muna Mergani and Pascal Bucher for invaluable help, advice and patience in the lab. This project was funded by ETH Zurich Research Grant, ETH-49 15-1 and Innosuisse, Bern, Switzerland, 32078.1 IP-LS.

\section{References}

Alenisan M.A., Alqattan H.H., Tolbah L.S., Shori A.B., 2017. Antioxidant properties of dairy products fortified with natural additives: A review. J. Assn. Arab. Univ. Basic Appl. Sci. 24, 101-106, https://doi.org/10.1016/j.jaubas.2017.05.001

Białek M., Czauderna M., Białek A., 2017. Conjugated linolenic acid (CLnA) isomers as new bioactive lipid compounds in ruminant-derived food products. A review. J. Anim Feed. Sci. 26, 354-358, https://doi.org/10.22358/jafs/68862/2017

Calder P., 2015. Functional roles of fatty acids and their effects on human health. J. Parenteral Enteral Nutr. 39, S1, 18S-32S, https://doi.org/10.1177/0148607115595980

Castro T., Martinez D., Isabel B., Cabezas A., Jimeno V., 2019. Vegetable oils rich in polyunsaturated fatty acids supplementation of dairy cows' diets: Effects on productive and reproductive performance. Animals 9, 205, https://doi. org/10.3390/ani9050205 
Chilliard Y., Glasser F., Ferlay A., Bernard L., Rouel J., Doreau M., 2007. Diet, rumen biohydrogenation and nutritional quality of cow and goat milk fat. Eur. J. Lipid Sci. Technol. 109, 82-55, https://doi.org/10.1002/ejlt.200700080

Collomb M., Bühler T., 2000. Analysis of the fatty acid composition of milk fat. Optimisation and validation of a general high resolution method (in French). Travaux de Chimie Alimentaire et d'Hygiène 91, 306-332

Denninger T.M., Schwarm A., Birkinshaw A. et al., 2020. Immediate effect of Acacia mearnsii tannins on methane emissions and milk fatty acid profiles of dairy cows. Anim. Feed Sci. Technol. 261, 114338, https://doi.org/10.1016/j. anifeedsci.2019.114388

Frutos P., Hervás G., Giráldez F.J., Mantecón A.R., 2004. Review. Tannins and ruminant nutrition. Span. J. Agric. Res. 2, 191-202, https://doi.org/10.5424/sjar/2004022-73

German J.B., Dillard C.J., 2006. Composition, structure and absorption of milk lipids: A source of energy, fat-soluble nutrients and bioactive molecules. Crit. Rev. Food Sci. Nutr. 46, 57-92, https://doi.org/10.1080/10408690590957098

Hanuš O., Samková E., Kř̌̌žová L., Hasoňová L., Kala R., 2018. Role of fatty acids in milk fat and the influence of selected factors on their variability - A review. Molecules 23, 1636, https://doi. org/10.3390/molecules23071636

IUPAC, 1987. Standard Methods for Analysis of Oils, Fats and Derivatives in Report of IUPAC Working Group WG 2/87. 7th Edition. Blackwell Scientific Publications. Oxford (UK)

Jayanegara A., Wina E., Soliva C.R., Marquardt S., Kreuzer M., Leiber F., 2011. Dependence of forage quality and methanogenic potential of tropical plants on their phenolic fractions as determined by principal component analysis. Anim. Feed Sci. Technol. 163, 231-243, https://doi.org/10.1016/j. anifeedsci.2010.11.009

Khiaosa-Ard R., Bryner S.F., Scheeder M.R.L., Wettstein H-R., Leiber F., Kreuzer M., Soliva C.R., 2009. Evidence for the inhibition of the terminal step of ruminal a-linolenic acid biohydrogenation by condensed tannins. J. Dairy Sci. 92, 177-188, https://doi. org/10.3168/jds.2008-1117

Leparmarai P.T., Sinz S., Liesegang A., Ortmann S., Kreuzer M., Marquardt S., 2019. Transfer of total phenols from a grapeseed supplemented diet to dairy sheep and goat milk, and effects on performance and milk quality. J. Anim. Sci. 97, 1840-1851, https://doi.org/10.1093/jas/skz046

Lourenço M., Ramos-Morales E., Wallace R.J., 2010. The role of microbes in rumen lipolysis and biohydrogenation and their manipulation. Animal 4, 1008-1023, https://doi.org/10.1017/ S175173111000042X

Majewska P., Kowalik B., 2020. Growth performance, carcass characteristics, fatty acid composition, and blood biochemical parameters of lamb fed diet with the addition of lingonberry leaves and oak bark. Eur. J. Lipid Sci. Technol. 122, 1900273, https://doi.org/10.1002/ejtt.201900273

Makkar H.P.S., 2003. Quantification of Tannin Measurement in Tree and Shrub Foliage: A Laboratory Manual. Kluwer Academic Publishers. Dordrecht (the Netherlands)

Månsson H.L., 2008. Fatty acids in bovine milk fat. Food Nutr. Res. 52, https://doi.org/10.3402/fnr.v52i0.1821
Morales R., Ungerfeld E.M., 2015. Use of tannins to improve fatty acids profile of meat and milk quality in ruminants: A review. Chilean J. Agric. Res. 75, 239-248, https://doi.org/10.4067/ S0718-58392015000200014

Palmquist D.R., Jenkins T.C., 2017. A 100 year review: fat feeding of dairy cows. J. Dairy Sci. 100, 10061-10077, https://doi. org/10.3168/jds.2017-12924

Petit H.V., Dewhurst R.J., Scollan N.D., Proulx J.D., Khalid M., Haresign W., Twagiramungu H., Mann G.E., 2002. Milk production and composition, ovarian function and prostaglandin secretion of dairy cows fed omega-3 fats. J. Dairy Sci. 85, 889-899, https://doi.org/10.3168/jds.S0022-0302(02)74147-7

Singh A., Nayak S., Baghel R.P.S., Khare A., Malapure C.D., Thakur D.S, Sharma P., Singh B.P., 2018. Dietary manipulations to alter milk fat composition. J. Entomol. Zool. 6, 176-181

Soberon A., Cherney J.H., Liu R.H., Ross D.A., Cherney D.J.R., 2012. Free ferulic acid uptake in lactating cows. J. Dairy Sci. 95, 6563-6570, https://doi.org/10.3168/jds.2011-5018

Suter B., Grob K., Pacciarelli B., 1997. Determination of fat content and fatty acid composition though 1-min transesterification in the food sample: principles. Z. Lebensm Unters Forsch A 204, 252-258, https://doi.org/10.1007/s002170050073

Terranova M., Wang S., Eggerschwiler L., Braun U., Kreuzer M., Schwarm A., 2020. Dose-response effect of woody and herbaceous forage plants to in vitro ruminal methane and ammonia formation, and their short-term palatability in lactating cows. Animal 14, 538-548, https://doi.org/10.1017/ S1751731119002076

Vasta V., Daghio M., Cappucci A., Buccioni A., Serra A., Vitti C., Mele M., 2019. Invited review: Plant polyphenols and rumen microbiota responsible for fatty acid biohydrogenation, fiber digestion, and methane emission: Experimental evidence and methodological approaches. J. Dairy Sci. 102, 3781-3804, https://doi.org/10.3168/jds.2018-14985

Vasta V., Mele M., Serra A., Scerra M., Luciano G., Lanza M., Priolo A., 2009. Metabolic fate of fatty acids involved in ruminal biohydrogenation in sheep fed concentrate or herbage with or without tannins. J. Anim. Sci. 87, 2674-2684, https://doi.org/10.2527/jas.2008-1761

Vázquez C.V., Rojas M.G.V., Ramírez C.A. et al., 2015. Total phenolic compounds in milk from different species. Design of an extraction technique for quantification using the FolinCiocalteu method. Food Chem. 176, 480-486, https://doi. org/10.1016/j.foodchem.2014.12.050

Vuazour D., Rodriguez-Mateous A., Corona G., Oruna-Concha M.J., Spencer J.P.E., 2010. Polyphenols and human health: Prevention of disease and mechanisms of action. Nutrients 2, 1106-1131, https://doi.org/10.3390/nu2111106

Wettstein H-R., Scheeder M.R.L., Sutter F., Kreuzer M., 2001. Effect of lecithins partly replacing rumen-protected fat on fatty acid digestion and composition of cow milk. Eur. J. Lipid Sci. Technol. 103, 12-22, https://doi.org/10.1002/14389312(200101)103:1<12::AID-EJLT12>3.0.CO;2-X

Willcox J.K., Ash S.L., Catignani G.L., 2004. Antioxidants and prevention of chronic disease. Crit. Rev. Food Sci. Nutr. 44, 275-295, https://doi.org/10.1080/1040869049046848 